\title{
IRRATIONALITY MEASURES FOR CUBIC IRRATIONALS WHOSE CONJUGATES LIE ON A CURVE
}

\author{
F. AMOROSO AND U. ZANNIER
}

\section{INTRODUCTION}

This paper deals with irrationality measure of algebraic irrational numbers. There is a vast bibliography on this subject, the interested reader can refer for instance to [8] for further references. Let us recall only some basic facts. We say that $\mu$ is an irrationality measure for $\xi \in \mathbb{R} \backslash \mathbb{Q}$ if for every $\varepsilon>0$ there exists $C_{\varepsilon}>0$ such that for all $p, q \in \mathbb{Z}$ with $q>0$ we have

$$
\left|\xi-\frac{p}{q}\right|>\frac{C_{\varepsilon}}{q^{\mu+\varepsilon}} .
$$

We define the irrationality measure $\mu(\xi)$ as the infimum of the irrationality measures for $\xi$. By Dirichlet's Theorem, or using the theory of continued fractions, $\mu(\xi) \geq 2$ and moreover for almost all real numbers equality holds. Nevertheless, 'few' examples of numbers with (provable) irrationality measure 2 are known.

For real irrational algebraic numbers the situation is different: by a celebrated result of Roth, which extends the Thue-Gelfond-Dyson-Siegel method from 2 to $n$ variables, every such number has irrationality measure 2, but this result is not effective, in the sense that the above $C_{\varepsilon}$ cannot be effectively computed (at least for small $\varepsilon>0$ ).

Let us say that $\mu$ is an effective irrationality measure for $\xi$, if the real number $C_{\varepsilon}$ above is effectively computable for any $\varepsilon>0$, and define $\mu_{\text {eff }}(\xi)$ as the infimum of the effective irrationality measures for $\xi$. Then Liouville's Theorem provides the easy bound $\mu_{\mathrm{eff}}(\xi) \leq d$ for a real algebraic number of degree $d>1$.

Feldman [12], using Baker's theory of linear forms in logarithms, proves that $\mu_{\mathrm{eff}}(\xi)<d$ for a real algebraic number of degree $d \geq 3$. Bombieri [6] gives another proof of this result, using the original Thue-Dyson-Siegel method in two variables.

In some special case, essentially when $\xi$ is "close" to 1 , the theory of Padé approximant can give much better result. For instance for $\xi:=\sqrt[3]{2}$, Baker [3] obtains in particular $\mu_{\text {eff }}(\xi) \leq 2.955$, using $\frac{4}{5} \sqrt[3]{2} \approx 1.008$.

The aim of this short paper is to describe an apparently new method to give good effective irrationality measures for certain algebraic irrational numbers.

In section 2 we shall first consider the special case, but still interesting in its own, of cubic roots $\xi_{l}=\sqrt[3]{l^{3}+1}$ where $l$ is a large natural number. The classical method deals with Padé approximants to the function $z \mapsto$ $(1-z)^{1-1 / 3}$. The main ingredient of our approach, which is easily illustrated

Date: February 20, 2019. 
by the example in section 2 below, is a bounded height estimate of Beukers and Schlickewei [4] for the solutions of the system of equations

$$
\begin{aligned}
A+B & =1 ; \\
a A^{n}+b B^{n} & =1 .
\end{aligned}
$$

in the unknown $A, B \in \overline{\mathbb{Q}}^{*}$, with coefficients $a, b \in \overline{\mathbb{Q}}^{*}$. Curiously enough, this estimate is a consequence of another Padé construction, those of the Padé approximants to the function $z \mapsto(1-z)^{n}$ (via hypergeometric functions).

In section 4 we generalize this approach to other cubic irrationals whose algebraic conjugates are coordinates of a point lying in a fixed line of $\mathbb{P}_{2}$.

In some cases, our results are close to the results obtained with the classical method [1]. In other cases they appears to be new. Of course, as in the classical case, refinements using some variant of the so-called Chudnowski method could be possible.

To go further, it seems that the result of [4] is no longer sufficient and also any methods based on explicit Padé approximants. We use instead, in section 5, a (very special) case of a recent bounded height result [2] obtained by Masser and the authors of this article to deal with cubic irrationals whose conjugates lie on an arbitrary curve. This allow us to prove (section 5) the following theorem, which we state here only for algebraic integers, to avoid technical definitions on heights (section 3). See Theorem 5.2 for the more general statement.

Theorem 1.1. Let $\mathbb{K} \subseteq \mathbb{C}$ be a real cubic number field; denote by $\sigma_{1}=$ $\mathrm{Id}, \sigma_{2}, \sigma_{3}$ the immersions $\mathbb{K} \hookrightarrow \mathbb{C}$. Let $\mathcal{C} \subset \mathbb{P}_{2}$ be a projective curve defined over $\overline{\mathbb{Q}}$. Let $\hbar>0, \lambda>1 / 2$ and $\varepsilon \in(0,1)$ such that

$$
\hbar \geq c^{2} \min \left(1, \frac{(\lambda-1 / 2)^{2}}{8(\lambda+1)} \varepsilon\right)^{-2}
$$

where $c=c(\mathcal{C})$ is an effective constant. Let $\theta \in \mathcal{O}_{\mathbb{K}}$ with conjugate $\theta_{1}=\theta$, $\theta_{2}, \theta_{3}$. We assume:

$$
\left(\theta_{1}: \theta_{2}: \theta_{3}\right) \in \mathcal{C}(\overline{\mathbb{Q}})
$$

and

$$
\max \left(\left|\theta_{2}\right|,\left|\theta_{3}\right|\right) \leq \hbar, \quad \log |\theta| \leq-\lambda \hbar
$$

Then

$$
\mu_{\text {eff }}(\xi) \leq \frac{\lambda+1}{\lambda-1 / 2}
$$

for every generator $\xi$ of $\mathbb{K}$.

As a corollary, we recover a (apparently not well known) result of Chudnwski [9] on irrationality measures for the values of a cubic algebraic function holomorphic at 0 (see Corollary 5.4).

In principle our method, could be further generalized to irrational numbers of higher degree, at the price of a technical extension of the relevant result of [2]. 


\section{An Example}

Let $l$ be a positive (large) integer. We look for an irrationality measure of the cubic irrational $\xi_{l}=\sqrt[3]{l^{3}+1}$. To illustrate our method, we want to show that $\mu_{\mathrm{eff}}\left(\xi_{l}\right) \rightarrow 2$ when $l \rightarrow \infty$ (which is of course a well-known result). Put

$$
\theta=\xi_{l}-l, \quad \zeta=\exp (2 \pi i / 3) .
$$

We denote by $\theta_{1}=\theta, \theta_{2}=\zeta \xi_{l}-l$ and $\theta_{3}=\zeta^{2} \xi_{l}-l$ the algebraic conjugates of $\theta$ and we write $\boldsymbol{\theta}=\left(\theta_{1}, \theta_{2}, \theta_{3}\right)$. Let

$$
\theta^{n}=\lambda_{n, 0}+\lambda_{n, 1} \theta+\lambda_{n, 2} \theta^{2}
$$

with $\boldsymbol{\lambda}_{n}=\left(\lambda_{n, 0}, \lambda_{n, 1}, \lambda_{n, 2}\right) \in \mathbb{Z}^{3}$. Thus $\boldsymbol{\theta}^{n}=\Theta \lambda_{n}$, where

$$
\Theta=\left(\begin{array}{lll}
1 & \theta_{1} & \theta_{1}^{2} \\
1 & \theta_{2} & \theta_{2}^{2} \\
1 & \theta_{3} & \theta_{3}^{2}
\end{array}\right) .
$$

Solving in $\boldsymbol{\lambda}_{n}$ the system, we find $\boldsymbol{\lambda}_{n}=\Theta^{-1} \boldsymbol{\theta}^{n}$. We have $\theta_{1} \theta_{2} \theta_{3}=1$ and $\left|\theta_{2}\right|=\left|\theta_{3}\right|=O(l)$ as $l \rightarrow+\infty$. Thus

$$
|\theta|=O\left(l^{-2}\right), \quad\left|\boldsymbol{\lambda}_{n}\right|^{1 / n} \ll l
$$

and the projective (non logarithmic) Weil height of $\boldsymbol{\theta} \in \mathbb{P}_{2}(\overline{\mathbb{Q}})$ satisfies

$$
H(\boldsymbol{\theta})=\left|\theta_{2}\right|=\left|\theta_{3}\right|=O(l) .
$$

The implicit constants are absolute, for $n$ sufficiently large with respect to $l$. In order to apply a standard irrationality criterium, we search "small" vectors $\mathbf{u}_{n}=\left(u_{n, 0}, u_{n, 1}, u_{n, 2}\right) \in \mathbb{Z}^{3}$ such that $\alpha_{n}=u_{n, 0}+u_{n, 1} \theta+u_{n, 2} \theta^{2}$ satisfies $^{1}$

$$
\alpha_{n} \theta^{n} \in \mathbb{Z}+\mathbb{Z} \theta .
$$

By (2.1), this holds if and only if $\mathbf{u}_{n}=\left(u_{n, 0}, u_{n, 1}, u_{n, 2}\right)$ is orthogonal to the vector

$$
\mathbf{v}_{n}=\left(\lambda_{n, 2}, \lambda_{n+1,2}, \lambda_{n+2,2}\right) \text {. }
$$

of norm $\left|\mathbf{v}_{n}\right|^{1 / n} \ll l$. By Minkowski's theorem we can find two linearly independent vectors $\mathbf{u}_{n}, \mathbf{u}_{n}^{\prime} \in \mathbf{v}_{n}^{\perp}$ such that $\left|\mathbf{u}_{n}\right| \leq\left|\mathbf{u}_{n}^{\prime}\right|$ and

$$
\left|\mathbf{u}_{n}\right|^{1 / n} \cdot\left|\mathbf{u}_{n}^{\prime}\right|^{1 / n} \ll\left|\mathbf{v}_{n}\right|^{1 / n} \ll l .
$$

Thus $\left|\mathbf{u}_{n}\right|^{1 / n} \ll l^{1 / 2}$. This gives a sequence $\left\{p_{n} / q_{n}\right\}_{n}$ of good rational approximations to $\theta$, defined by $\left(u_{n, 0}+u_{n, 1} \theta+u_{n, 2} \theta^{2}\right) \theta^{n}=q_{n} \theta-p_{n}$. In fact, by $(2.1)$ and (2.2) we have

$$
\begin{aligned}
\left|q_{n}\right|^{1 / n} & \ll\left|\mathbf{u}_{n}\right|^{1 / n}\left|\boldsymbol{\lambda}_{n}\right|^{1 / n} \ll l^{3 / 2} \\
\left|q_{n} \theta-p_{n}\right|^{1 / n} & \ll\left|\mathbf{u}_{n}\right|^{1 / n}|\theta| \ll l^{-3 / 2}
\end{aligned}
$$

Let us assume that $\left|\mathbf{u}_{n}^{\prime}\right|$ satisfies a similar upper bound as $\left|\mathbf{u}_{n}\right|$, that is $\left|\mathbf{u}_{n}^{\prime}\right|^{1 / n} \ll l^{1 / 2}$. By (2.4) this holds if (and only if) the previous upper bound for $\left|\mathbf{u}_{n}\right|$ is essentially optimal, i.e.

$$
\left|\mathbf{u}_{n}\right|^{1 / n} \gg l^{1 / 2} .
$$

\footnotetext{
${ }^{1} \mathrm{~A}$ similar requirement was worked by Bombieri and Van der Poorten for a different pourpose.
} 
Note that in general this is what one would expect. Then, corresponding to $u_{n}^{\prime}$, one has another sequence $\left\{p_{n}^{\prime} / q_{n}^{\prime}\right\}_{n}$ of good rational approximations such that $p_{n} q_{n}^{\prime} \neq p_{n}^{\prime} q_{n}$ for all $n$. This would allow to conclude (by a standard irrationality criterion) that

$$
\mu_{\mathrm{eff}}\left(\xi_{l}\right) \rightarrow 2 \text { as } l \rightarrow+\infty
$$

Hence the success of this approach is reduced to producing a lower bound for $\left|\mathbf{u}_{n}\right|^{1 / n}$. Replacing $n$ by $2 n$, we can assume $n$ even. For $j=1,2,3$ write $\alpha_{n, j}=u_{n, 0}+u_{n, 1} \theta_{j}+u_{n, 2} \theta_{j}^{2}$. Since the height of $\boldsymbol{\alpha}_{n} \in \mathbb{P}_{2}(\overline{\mathbb{Q}})$ satisfies

$$
H\left(\boldsymbol{\alpha}_{n}\right)^{1 / n} \ll\left|\mathbf{u}_{n}\right|^{1 / n},
$$

it is enough to get a lower bound for this height.

Remark 2.1. For $j=1,2,3$ let $\sigma_{j}: \mathbb{Q}(\theta) \rightarrow \mathbb{C}$ be the immersions given by $\sigma_{j} \theta=\theta_{j}$. Then for $\gamma \in \mathbb{Z}[\theta]$ we have $\gamma \in \mathbb{Z}+\mathbb{Z} \theta$ if and only if $\sigma_{1} \gamma+\zeta \sigma_{2} \gamma+$ $\zeta^{2} \sigma_{3} \gamma=0$.

Since both $\theta$ and $\alpha_{n, 1} \theta^{n}$ are in $\mathbb{Z}+\mathbb{Z} \theta$, we have the two equations:

$$
\begin{aligned}
\theta_{1}+\zeta \theta_{2}+\zeta^{2} \theta_{3} & =0 ; \\
\alpha_{n, 1} \theta_{1}^{n}+\zeta \alpha_{n, 2} \theta_{2}^{n}+\zeta^{2} \alpha_{n, 3} \theta_{3}^{n} & =0 .
\end{aligned}
$$

We now use the following result of Beukers and Schlickewei ([4], lemme 2.3 ), which follows from an explicit construction ([5], lemma 6) of Padé approximants to the function $z \mapsto(1-z)^{n}$.

Lemma 2.2 (Beukers-Schlickewei). Let $a, b, A, B \in \overline{\mathbb{Q}}^{*}$ such that

$$
\begin{aligned}
A+B & =1 ; \\
a A^{n}+b B^{n} & =1
\end{aligned}
$$

for some even integer $n \in \mathbb{N}$. Then, $H(1: A: B) \leq c 2^{2 / n} H(1: a: b)^{2 / n}$, where $c=6 \sqrt{3}$.

The system (2.7) transforms into (2.8) by the change of variables:

$$
A=-\frac{\zeta \theta_{2}}{\theta_{1}}, \quad B=-\frac{\zeta^{2} \theta_{3}}{\theta_{1}}, \quad a=-\frac{\zeta \alpha_{n, 2}}{\zeta^{n} \alpha_{n, 1}}, \quad b=-\frac{\zeta^{2} \alpha_{n, 3}}{\zeta^{2 n} \alpha_{n, 1}} .
$$

We have

$$
H(1: A: B)=H(\boldsymbol{\theta}) \quad \text { and } \quad H(1: a: b)=H\left(\boldsymbol{\alpha}_{n}\right) .
$$

By the quoted result of Beukers and Schlickewei, by (2.3) and by (2.6),

$$
l \ll H(\boldsymbol{\theta}) \leq c 2^{2 / n} H\left(\boldsymbol{\alpha}_{n}\right)^{2 / n} \ll\left|\mathbf{u}_{n}\right|^{2 / n} .
$$

Thus (2.5) holds and

$$
\mu_{\text {eff }}\left(\xi_{l}\right) \rightarrow 2
$$

as claimed. 
Of course we can quantify all of this to see how near we go to effective irrationality measure 2. Assume $l \geq 3$. A more precise computation (see the first example 4.3) shows that $\xi_{l}$ has irrationality measure

$$
\begin{aligned}
\mu_{\mathrm{eff}}\left(\xi_{l}\right) & \leq 1+\frac{-\log \left(\sqrt[3]{l^{3}+1}-l\right)+\frac{2}{3} \log (6 \sqrt{3})}{-\log \left(\sqrt[3]{l^{3}+1}-l\right)-\frac{2}{3} \log (6 \sqrt{3})} \\
& =2+\frac{2 \log (6 \sqrt{3})}{3 \log l}+O\left((\log l)^{-2}\right) \text { for } l \rightarrow+\infty .
\end{aligned}
$$

Our method rests essentially on an explicit Padé construction (via hypergeometric function) of the Padé approximants to the function $z \mapsto(1-z)^{n}$, which is the main tool of the proof of Lemma 2.2. Let us compare our results with (a special case) of a result of Alladi and Robinson [1] which uses Padé approximants to the function $z \mapsto(1-z)^{1-1 / 3}$. Theorem 2 of op.cit. (with $\left.k=3, f(3)=9 / 4, r=1, s=l^{3}\right)$ gives, again for $l \geq 3$,

$$
\begin{aligned}
\mu_{\mathrm{eff}}\left(\xi_{l}\right) & \leq 1+\frac{-\log \left(\sqrt{l^{3}+1}-\sqrt{l^{3}}\right)+9 / 4}{-\log \left(\sqrt{l^{3}+1}-\sqrt{l^{3}}\right)-9 / 4} \\
& =2+\frac{3}{2 \log l}+O\left((\log l)^{-2}\right) \text { for } l \rightarrow+\infty .
\end{aligned}
$$

Since $2 \log (6 \sqrt{3}) / 3=1.567 \ldots>3 / 2$, the result of Alladi and Robisons is slightly better for large $l$. For small values of $l$ we obtain:

$$
\begin{array}{ll}
l=2 & \mu_{\text {eff }}=5.2381 \\
l=3 & \mu_{\text {eff }}=3.7865 \\
l=4 & \mu_{\text {eff }}=3.3480 \\
l=5 & \mu_{\text {eff }}=3.1312 \\
l=6 & \mu_{\text {eff }}=2.9996 \\
l=7 & \mu_{\text {eff }}=2.9099 \\
l=8 & \mu_{\text {eff }}=2.8443 \\
l=9 & \mu_{\text {eff }}=2.7937
\end{array}
$$

where we have reproduced the corresponding results of [1] in brackets. Note that our method gives something better for $l \leq 5$. But in these cases our effective irrationality measure is worse than the bound $\mu_{\text {eff }}\left(\xi_{l}\right) \leq 3$ predicted by Liouville's Theorem.

\section{Heights And absolute values.}

To go further, we need some more notation. As we have seen in section 2, given a cubic irrational $\theta$ the natural height associated with our method is not the Weil height of $\theta$ but instead the height of the projective point defined by its conjugates. Moreover, if $\theta$ is not a unit (as it was in section 2), for its absolute value we have to take into account the contribution of the finite places. We develop these remarks in the more general setting of algebraic numbers of degree $r$ in view of further applications. 
We consider algebraic numbers as complex numbers, on choosing an immersion $\overline{\mathbb{Q}} \hookrightarrow \mathbb{C}$. Given $\theta_{1}, \ldots, \theta_{r} \in \overline{\mathbb{Q}}$ we denote by $H(\boldsymbol{\theta})$ the normalized, non-logarithmic Weil's height of $\boldsymbol{\theta}=\left(\theta_{1}, \cdots, \theta_{r}\right)$, which we identify with the corresponding point in $\mathbb{P}_{r-1}(\overline{\mathbb{Q}})$. We put $h(\boldsymbol{\theta})=\log H(\boldsymbol{\theta})$ for the corresponding logarithmic height. For an algebraic number $\theta$ we set $H(\theta)=H(1: \theta)$ and $h(\theta)=\log H(\theta)$.

Let $\theta \in \mathbb{C}$ be an algebraic number. We choose a number field $\mathbb{K} \subseteq \mathbb{C}$ of degree say $r$, which contains $\theta$, we let $\mathbb{L}$ be any number field containing its Galois closure, and we denote $\sigma_{1}, \ldots, \sigma_{r}$ the immersions $\mathbb{K} \hookrightarrow \mathbb{C}$. We set

$$
H_{0}(\theta)=H\left(\sigma_{1} \theta: \cdots: \sigma_{r} \theta\right), \quad h_{0}(\theta)=\log H_{0}(\theta),
$$

for the Weil height of the projective point $\left(\sigma_{1} \theta: \cdots: \sigma_{r} \theta\right)$, and we let

$$
\|\theta\|=|\theta| \cdot H_{\text {finite }}(\theta)
$$

where

$$
H_{\text {finite }}(\theta):=\prod_{\substack{v \in \mathcal{M}_{\mathbb{L}} \\ v \nmid \infty}} \max \left(\left|\sigma_{1} \theta\right|_{v}, \ldots,\left|\sigma_{r} \theta\right|_{v}\right)^{\left[\mathbb{L}_{v}: \mathbb{Q}_{v}\right] /[\mathbb{L}: \mathbb{Q}]} .
$$

It is clear that these definitions do not depend on the choice of $\mathbb{L}$ and $\mathbb{K}$ (but the definition of $\|\theta\|$ depends on the immersion $\overline{\mathbb{Q}} \hookrightarrow \mathbb{C}$ ). We also remark that $H_{\text {finite }}(\theta) \leq 1$ if $\theta$ is an algebraic integer, with equality if $\theta$ is a unit.

Given the minimal polynomial of $\theta$, say

$$
x^{r}-a_{1} x^{r-1}+\cdots+(-1)^{r} a_{r} \in \mathbb{Q}[x],
$$

it easy to compute $H_{0}(\cdot)$ and $\|\cdot\|$. First

$$
\prod_{\substack{v \in \mathcal{M}_{\mathbb{L}} \\ v \mid \infty}} \max \left(\left|\sigma_{1} \theta\right|_{v}, \ldots,\left|\sigma_{r} \theta\right|_{v}\right)^{\left[\mathbb{L}_{v}: \mathbb{Q}_{v}\right] /[\mathbb{L}: \mathbb{Q}]}=\max \left(\left|\sigma_{1} \theta\right|, \ldots,\left|\sigma_{r} \theta\right|\right) .
$$

Thus

$$
H_{0}(\theta)=\max \left(\left|\sigma_{1} \theta\right|, \ldots,\left|\sigma_{r} \theta\right|\right) H_{\text {finite }}(\theta), \quad\|\theta\|=|\theta| H_{\text {finite }}(\theta) .
$$

The computation of $H_{\text {finite }}(\theta)$ is a little bit more involved.

Lemma 3.1. Let $v \in \mathcal{M}_{\mathbb{L}}, v \nmid \infty$. Then

$$
\max \left(\left|\sigma_{1} \theta\right|_{v}, \ldots,\left|\sigma_{r} \theta\right|_{v}\right)=\max \left(\left|a_{1}\right|_{v},\left|a_{2}\right|_{v}^{1 / 2}, \ldots,\left|a_{r}\right|_{v}^{1 / r}\right) .
$$

Proof. Let for short $\theta_{j}:=\sigma_{j} \theta$ and $\Theta=\max \left(\left|\theta_{1}\right|_{v}, \ldots,\left|\theta_{r}\right|_{v}\right)$. After renumbering we can assume

$$
\left|\theta_{1}\right|_{v}=\cdots=\left|\theta_{k}\right|_{v}>\left|\theta_{k+1}\right|_{v} \geq \cdots \geq\left|\theta_{r}\right|_{v}
$$

for some $k$ with $1 \leq k \leq r$. Then obviously

$$
\left|a_{j}\right|_{v}=\left|\sum_{1 \leq i_{1}<\cdots<i_{j} \leq r} \theta_{i_{1}} \cdots \theta_{i_{j}}\right|_{v} \leq \Theta^{j}
$$

for $j=1, \ldots, r$. Moreover $\left|a_{k}\right|_{v}=\Theta^{k}$. Indeed, let $i_{1}, \ldots, i_{k}$ with $1 \leq i_{1}<$ $\cdots<i_{k} \leq r$. Then, by $(3.1)$

$$
\left|\theta_{i_{1}} \cdots \theta_{i_{k}}\right|_{v}<\left|\theta_{1} \cdots \theta_{k}\right|_{v}=\Theta^{k}
$$


if $\left(i_{1}, \ldots, i_{k}\right) \neq(1, \ldots, k)$.

Thus

$$
H_{\text {finite }}(\theta)=\prod_{p} \max \left(\left|a_{1}\right|_{p},\left|a_{2}\right|_{p}^{1 / 2}, \ldots,\left|a_{r}\right|_{p}^{1 / r}\right),
$$

where the product is over the (finite) rational primes.

Even more explicitly, assume $r=3$ (which is the relevant case for this article) and let

$$
x^{3}+f_{1} x^{2}+f_{2} x+f_{3} \in \mathbb{Q}[x]
$$

with $\operatorname{root} \theta$. Then

$$
H_{\text {finite }}(\theta)=\operatorname{lcm}\left(\operatorname{den}\left(f_{1}\right)^{6}, \operatorname{den}\left(f_{2}\right)^{3}, \operatorname{den}\left(f_{3}\right)^{2}\right)^{1 / 6} .
$$

We also remark that (by Remark 3.21 ) below) we may assume $f_{1}=1$ replacing $\theta$ by $f_{1} \theta$.

We now state some obvious properties of $H_{0}(\cdot)$ and $\|\cdot\|$.

\section{Remark 3.2.}

1) $H_{0}(\cdot)$ and $\|\cdot\|$ are functions $\overline{\mathbb{Q}} \rightarrow \mathbb{R}^{+}$invariant by multiplication by non-zero rational numbers.

2) For $\theta \in \overline{\mathbb{Q}}$ of degree $r$ we have $\|\theta\| \leq H_{0}(\theta) \leq H(\theta)^{r}$ and $\|\theta\| \leq|\theta| \cdot H(\theta)^{r}$.

3) For $\theta, \theta^{\prime} \in \overline{\mathbb{Q}}$ we have $H_{0}\left(\theta \cdot \theta^{\prime}\right) \leq H_{0}(\theta) \cdot H_{0}\left(\theta^{\prime}\right)$ and $\left\|\theta \cdot \theta^{\prime}\right\| \leq\|\theta\| \cdot\left\|\theta^{\prime}\right\|$.

We state below a variant of Liouville's inequality:

Remark 3.3. Let $\theta$ be a non zero algebraic number of degree $r$. Then

$$
\|\theta\| \geq H_{0}(\theta)^{-(r-1)} .
$$

We also need a variant of a classical irrationality measures criterium. We start with

Lemma 3.4. Let $\xi \in \overline{\mathbb{Q}}$ and $q_{0}, \ldots, q_{r-1} \in \mathbb{Q}$. Put

$$
\theta=q_{0}+q_{1} \xi+\cdots+q_{r-1} \xi^{r-1} .
$$

Then

$$
H_{0}(\theta) \leq r H(\xi)^{r(r-1)} \cdot H(\mathbf{q}) \text { and }\|\theta\| \leq H(\xi)^{r(r-1)} d(\mathbf{q}) \cdot|\theta|
$$

with $d(\mathbf{q})=\prod_{p \text { prime }} \max \left(\left|q_{0}\right|_{p}, \ldots,\left|q_{r-1}\right|_{p}\right)$. Moreover, if $\xi$ has degree $r$,

$$
H(\mathbf{q}) \leq c_{1} H_{0}(\theta) \text { and } d(\mathbf{q}) \cdot|\theta| \leq c_{2}\|\theta\|
$$

for some positive constants $c_{1}$ and $c_{2}$ depending only on $\xi$.

Proof. We keep all the notations above and we let $\xi_{j}=\sigma_{j} \xi, \theta_{j}=\sigma_{j} \theta$. Let $v$ be a place of $\mathbb{L}$. Put $\varepsilon_{v}=1$ if $v \nmid \infty$ and $\varepsilon_{v}=0$ otherwise. Then

$$
\left|\theta_{j}\right|_{v} \leq r^{\varepsilon_{\nu}} \max \left(\left|q_{0}\right|_{v}, \ldots,\left|q_{r-1}\right|_{v}\right) \max \left(1,\left|\xi_{j}\right|_{v}\right)^{r-1}
$$


Thus

$$
\begin{aligned}
\max \left(\left|\theta_{1}\right|_{v}, \ldots,\left|\theta_{r}\right|_{v}\right) & \leq r^{\varepsilon_{\nu}} \max \left(\left|q_{0}\right|_{v}, \ldots,\left|q_{r-1}\right|_{v}\right) \max \left(1,\left|\xi_{1}\right|_{v}, \ldots,\left|\xi_{r}\right|_{v}\right)^{r-1} \\
& \leq r^{\varepsilon_{\nu}} \max \left(\left|q_{0}\right|_{v}, \ldots,\left|q_{r-1}\right|_{v}\right) \prod_{j=1}^{r} \max \left(1,\left|\xi_{j}\right|_{v}\right)^{r-1} .
\end{aligned}
$$

Inequalities (3.3) follow. To prove (3.4), we solve the system

$$
q_{0}+q_{1} \xi_{j}+\cdots+q_{r-1} \xi_{j}^{r-1}=\theta_{j}, \quad j=1, \ldots, r
$$

in the unknowns $q_{0}, \ldots, q_{r-1}$. We find

$$
\left(q_{0}, \ldots, q_{r-1}\right)=\Xi^{-1}\left(\theta_{1}, \ldots, \theta_{r}\right)^{T}
$$

where ${ }^{T}$ denote transposition and where $\Xi$ is the $r \times r$ matrix $\xi_{i}^{j-1}$ of determinant $\sqrt{|\operatorname{disc}(\xi)|}$. Thus

$$
\max \left(\left|q_{0}\right|_{v}, \ldots,\left|q_{r-1}\right|_{v}\right) \leq c_{v}(\xi) \max \left(\left|\theta_{1}\right|_{v}, \ldots,\left|\theta_{r}\right|_{v}\right)
$$

which gives the last inequalities.

We can now state our version of a classical irrationality measure criterium.

Lemma 3.5. Let $\xi$ be an algebraic number of degree $r$. Let also $s$ be a positive integer $\leq r$ and $\theta_{n}^{(i)}(i=1, \ldots, s)$ be sequences in

$$
\mathbb{Q}+\mathbb{Q} \xi+\cdots+\mathbb{Q} \xi^{s-1}
$$

such that $\theta_{n}^{(1)}, \ldots, \theta_{n}^{(s)}$ are $\mathbb{Q}$-linearly independent for large $n$.

Let us assume that there exist $H>1, n_{0} \in \mathbb{N}$ and $\omega>0$ such that, for $i=1, \ldots, s$ and for $n \geq n_{0}$

$$
H_{0}\left(\theta_{n}^{(i)}\right) \leq H^{n}, \quad\left\|\theta_{n}^{(i)}\right\| \leq H^{-\omega n} .
$$

Then $\xi$ has an effective irrationality measure $\leq(s-1)(1+1 / \omega)$.

Proof. We fix an index $i$. Multiplying $\theta_{n}^{(i)}$ by suitable rationals numbers, we may assume (by 1) of Remark 3.2)

$$
\theta_{n}^{(i)}=p_{n, 0}^{(i)}+p_{n, 1}^{(i)} \xi+\cdots+p_{n, s-1}^{(i)} \xi^{s-1}
$$

with $p_{n, 0}^{(i)}, \ldots, p_{n, s-1}^{(i)}$ coprime integers. Since $\theta_{n}^{(1)}, \ldots, \theta_{n}^{(s)}$ are $\mathbb{Q}$-linearly independent for large $n$, the vectors $\left(p_{n, 0}^{(i)}, \ldots, p_{n, s-1}^{(i)}\right)$ are also $\mathbb{Q}$-linearly independent. Moreover, by (3.4) of Lemma 3.4,

$$
\max \left(\left|p_{n, 0}^{(i)}\right|,\left|p_{n, 1}^{(i)}\right|, \ldots,\left|p_{n, s-1}^{(i)}\right|\right) \leq C H^{n}
$$

and

$$
\left|p_{n, 0}^{(i)}+p_{n, 1}^{(i)} \xi+\cdots+p_{n, s-1}^{(i)} \xi^{s-1}\right| \leq C H^{-\omega n}
$$

for some $C>0$ depending only on $\xi$. We now apply a standard irrationality criterium. 


\section{Conjugates lying ON a fiXed line.}

The computations in section 2 can be easily generalized to a (real) cubic irrational $\theta$ with algebraic conjugates $\theta_{1}, \theta_{2}, \theta_{3}$ defining a projective point in a fixed projective line. Moreover, the method gives an upper bound for the effective irrationality measure of an arbitrary generator of $\mathbb{Q}(\theta)$.

Let $L$ be a projective line in $\mathbb{P}_{2}$ defined over $\overline{\mathbb{Q}}$ by

$$
\beta_{1}^{-1} x_{1}+\beta_{2}^{-1} x_{2}+\beta_{3}^{-1} x_{3}=0 .
$$

Put $c(L)=(6 \sqrt{3})^{1 / 2} H(\boldsymbol{\beta})^{3 / 2}$.

Theorem 4.1. Let $\mathbb{K} \subseteq \mathbb{C}$ be a real cubic number field; denote by $\sigma_{1}=$ $\mathrm{Id}, \sigma_{2}, \sigma_{3}$ the immersions $\mathbb{K} \hookrightarrow \mathbb{C}$. Let $\theta \in \mathbb{K}$ such that

$$
\|\theta\|<c(L)^{-1} H_{0}(\theta)^{-1 / 2} .
$$

We assume:

$$
\left(\sigma_{1} \theta: \sigma_{2} \theta: \sigma_{3} \theta\right) \in L(\overline{\mathbb{Q}}) .
$$

Then every generator of $\mathbb{K}$ has irrationality measure

$$
\leq 1+\frac{\frac{3}{2} h_{0}(\theta)+\log c(L)}{\log (1 /\|\theta\|)-\frac{1}{2} h_{0}(\theta)-\log c(L)} .
$$

Proof. We first remark that $\theta$ is irrational by Remark 3.3 since $\|\theta\|<1$, and thus $\mathbb{K}=\mathbb{Q}(\theta)$. Let $\theta_{j}=\sigma_{j} \theta$. We then proceed as in section 2. For the reader's convenience we make all details explicit. Let $\xi$ be a generator of $\mathbb{Q}(\theta)$ with algebraic conjugates $\xi_{1}=\xi, \xi_{2}, \xi_{3}$. For $n \in \mathbb{N}$ write

$$
\theta^{n}=\lambda_{n, 0}+\lambda_{n, 1} \xi+\lambda_{n, 2} \xi^{2}
$$

with $\lambda_{n, j} \in \mathbb{Q}$. We look for "small" vectors $\mathbf{u}_{n}=\left(u_{n, 0}, u_{n, 1}, u_{n, 2}\right) \in \mathbb{Z}^{3}$ such that

$$
\left(u_{n, 0}+u_{n, 1} \theta+u_{n, 2} \theta^{2}\right) \theta^{n} \in \mathbb{Q}+\mathbb{Q} \xi .
$$

This holds if and only if $\mathbf{u}_{n}=\left(u_{n, 0}, u_{n, 1}, u_{n, 2}\right)$ is orthogonal to the vector $\mathbf{v}_{n}=\left(\lambda_{n, 2}, \lambda_{n+1,2}, \lambda_{n+2,2}\right)$. Solving the $9 \times 9$ system

$$
\lambda_{i, 0}+\lambda_{i, 1} \theta_{j}+\lambda_{i, 2} \theta_{j}^{2}=\theta_{j}^{i}, \quad i=n, n+1, n+2 ; j=1,2,3,
$$

we see that

$$
H\left(\mathbf{v}_{n}\right) \leq H\left(\lambda_{n, 0}: \lambda_{n, 1}: \cdots: \lambda_{n+2,2}\right) \ll H\left(\theta_{1}^{n}: \theta_{2}^{n}: \cdots: \theta_{3}^{n+2}\right),
$$

where from now on the implicit constants in $\ll$ may depend on $\boldsymbol{\xi}$ and on $\boldsymbol{\theta}$, but not on $n$. By standard computations,

$$
H\left(\theta_{1}^{n}: \theta_{2}^{n}: \cdots: \theta_{3}^{n+2}\right) \leq H\left(\theta_{1}: \theta_{2}: \theta_{3}\right)^{n} H\left(\theta_{1}\right)^{2} H\left(\theta_{2}\right)^{2} H\left(\theta_{3}\right)^{2} .
$$

Thus

$$
H\left(\mathbf{v}_{n}\right) \ll H_{0}(\theta)^{n} .
$$

By Minkowski's theorem we can find two linearly independent vector $\mathbf{u}_{n}$, $\mathbf{u}_{n}^{\prime} \in \mathbf{v}_{n}^{\perp}$ with rational entries such that

$$
H\left(\mathbf{u}_{n}\right) H\left(\mathbf{u}_{n}^{\prime}\right) \ll H\left(\mathbf{v}_{n}\right) \ll H_{0}(\theta)^{n} .
$$

Write

$$
\alpha_{n}=u_{n, 0}+u_{n, 1} \theta+u_{n, 2} \theta^{2}, \quad \alpha_{n}^{\prime}=u_{n, 0}^{\prime}+u_{n, 1}^{\prime} \theta+u_{n, 2}^{\prime} \theta^{2}
$$


Thus $\alpha_{n} \theta_{n}, \alpha_{n}^{\prime} \theta_{n}$ are $\mathbb{Q}$-linearly independent algebraic number in $\mathbb{Q}+\mathbb{Q} \xi$. To get an irrationality measure for $\theta$ via Lemma 3.5 we need an upper for $H_{0}\left(\alpha_{n} \theta_{n}\right),\left\|\alpha_{n} \theta_{n}\right\|$ and for the corresponding dashed quantities. By symmetry, it is enough to consider the last ones; we can also assume $n$ even. By 2) and 3) of Remark 3.2 we have

$$
H_{0}\left(\alpha_{n}^{\prime} \theta^{n}\right) \leq H_{0}\left(\alpha_{n}^{\prime}\right) H(\theta)^{n}, \quad\left\|\alpha_{n}^{\prime} \theta^{n}\right\| \leq H_{0}\left(\alpha_{n}^{\prime}\right) .
$$

By the first inequality in (3.3) of Lemma 3.4, $H_{0}\left(\alpha_{n}\right) \ll H\left(\mathbf{u}_{n}\right)$ and $H_{0}\left(\alpha_{n}^{\prime}\right) \ll$ $H\left(\mathbf{u}_{n}^{\prime}\right)$. Thus by $(4.2)$

$$
H_{0}\left(\alpha_{n}\right) H_{0}\left(\alpha_{n}^{\prime}\right) \ll H_{0}(\theta)^{n} .
$$

and

$$
\begin{aligned}
H_{0}\left(\alpha_{n}^{\prime} \theta^{n}\right) & \ll H_{0}\left(\alpha_{n}\right)^{-1} H_{0}(\theta)^{2 n} \\
\left\|\alpha_{n}^{\prime} \theta^{n}\right\| & \ll H_{0}\left(\alpha_{n}\right)^{-1} H_{0}(\theta)^{n}\|\theta\|^{n}
\end{aligned}
$$

As in section 2, we need a lower bound for $H_{0}\left(\alpha_{n}\right)$ (note that this represents the crucial issue). Put

$$
\alpha_{n, j}:=u_{n, 0}+u_{n, 1} \theta_{j}+u_{n, 2} \theta_{j}^{2} \quad \text { for } j=1,2,3 .
$$

We have $\boldsymbol{\theta} \in L$; thus

$$
\beta_{1}^{-1} \theta_{1}+\beta_{2}^{-1} \theta_{2}+\beta_{3}^{-1} \theta_{3}=0 .
$$

Moreover $\alpha_{n} \theta^{n} \in \mathbb{Q}+\mathbb{Q} \xi$ and thus

$$
\left(\alpha_{n, 1} \theta_{1}^{n}: \alpha_{n, 2} \theta_{2}^{n}: \alpha_{n, 3} \theta_{3}^{n}\right) \in L^{\prime},
$$

where $L^{\prime}$ is the line in $\mathbb{P}_{2}$ through the points $\mathbf{1}$ and $\boldsymbol{\xi}$, of equation

$$
\left|\begin{array}{ccc}
x_{1} & x_{2} & x_{3} \\
1 & 1 & 1 \\
\xi_{1} & \xi_{2} & \xi_{3}
\end{array}\right|=\xi_{1}^{\prime} x_{1}+\xi_{2}^{\prime} x_{2}+\xi_{3}^{\prime} x_{3}=0
$$

with $\xi^{\prime}=\left(\xi_{3}-\xi_{2}, \xi_{1}-\xi_{3}, \xi_{2}-\xi_{1}\right)$. Thus

$$
\xi_{1}^{\prime} \alpha_{n, 1} \theta_{1}^{n}+\xi_{2}^{\prime} \alpha_{n, 2} \theta_{2}^{n}+\xi_{3}^{\prime} \alpha_{n, 3} \theta_{3}^{n}=0 .
$$

Let

$$
A=-\beta_{2}^{-1} \theta_{2} / \beta_{1}^{-1} \theta_{1}, \quad B=-\beta_{3}^{-1} \theta_{3} / \beta_{1}^{-1} \theta_{1}
$$

and

$$
a=-\xi_{2}^{\prime} \alpha_{n, 2} \beta_{2}^{n} / \xi_{1}^{\prime} \alpha_{n, 1} \beta_{1}^{n}, \quad b=-\xi_{3}^{\prime} \alpha_{n, 3} \beta_{3}^{n} / \xi_{1}^{\prime} \alpha_{n, 1} \beta_{1}^{n} .
$$

Recall that we assume $n$ even. By (4.4) and (4.5) we have

$$
A+B=1, \quad a A^{2 \rho}+b B^{2 \rho}=1
$$

with $\rho=n / 2 \in \mathbb{N}$. By the already quoted [4], Lemma 2.3,

$$
H(1: A: B) \leq c 2^{2 / n} H(1: a: b)^{2 / n} .
$$

with $c=6 \sqrt{3}$. We have

$$
\begin{aligned}
H(1: A: B) & =H\left(\beta_{1}^{-1} \theta_{1}: \beta_{2}^{-1} \theta_{2}: \beta_{3}^{-1} \theta_{3}\right) \\
& \geq H(\boldsymbol{\beta})^{-1} H(\boldsymbol{\theta})=H(\boldsymbol{\beta})^{-1} H_{0}(\theta)
\end{aligned}
$$


and

$$
\begin{aligned}
H(1: a: b) & =H\left(\xi_{1}^{\prime} \alpha_{n, 1} \beta_{1}^{n}: \xi_{2}^{\prime} \alpha_{n, 2} \beta_{2}^{n}: \xi_{3}^{\prime} \alpha_{n, 3} \beta_{3}^{n}\right) \\
& \leq H\left(\boldsymbol{\xi}^{\prime}\right) H(\boldsymbol{\beta})^{n} H\left(\boldsymbol{\alpha}_{n}\right)=H\left(\boldsymbol{\xi}^{\prime}\right) H(\boldsymbol{\beta})^{n} H_{0}\left(\alpha_{n}\right)
\end{aligned}
$$

Thus

$$
H(\boldsymbol{\beta})^{-n / 2} H_{0}(\theta)^{n / 2} \leq 2 c^{n / 2} H\left(\boldsymbol{\xi}^{\prime}\right) H(\boldsymbol{\beta})^{n} H_{0}\left(\alpha_{n}\right) \ll c^{n / 2} H(\boldsymbol{\beta})^{n} H_{0}\left(\alpha_{n}\right)
$$

and finally

$$
H_{0}\left(\alpha_{n}\right)^{-1} \ll c^{n / 2} H(\boldsymbol{\beta})^{3 n / 2} H_{0}(\theta)^{-n / 2}=c(L)^{n} H_{0}(\theta)^{-n / 2}
$$

By (4.3),

$$
H_{0}\left(\alpha_{n}^{\prime} \theta^{n}\right) \ll H_{0}\left(\alpha_{n}\right)^{-1} H_{0}(\theta)^{2 n} \ll\left(c(L) H_{0}(\theta)^{3 / 2}\right)^{n}
$$

and

$$
\begin{aligned}
\left\|\alpha_{n}^{\prime} \theta^{n}\right\| \ll H_{0}\left(\alpha_{n}\right)^{-1} H_{0}(\theta)^{n}\|\theta\|^{n} & \ll\left(c(L) H_{0}(\theta)^{1 / 2}\|\theta\|\right)^{n} \\
& =\left(c(L) H_{0}(\theta)^{3 / 2}\right)^{-\omega n}
\end{aligned}
$$

with

$$
\omega=\frac{\log (1 /\|\theta\|)-\frac{1}{2} h_{0}(\theta)-\log c(L)}{\frac{3}{2} h_{0}(\theta)+\log c(L)} .
$$

Since $\omega>0$ by (4.1), Lemma 3.5 (with $r=3$ and $s=2$ ) shows that $\xi$ has irrationality measure $\leq 1+1 / \omega$.

Remark 4.2. We get non-trivial effective irrationality measures, i. e. better than 3 (Liouville's Theorem), if

$$
\|\theta\|<c(L)^{-3 / 4} H_{0}(\theta)^{-5 / 4} .
$$

We get effective irrationality measures $\leq 2+\varepsilon$ if

$$
\|\theta\| \leq c(L)^{-1+\varepsilon_{2}} H_{0}(\theta)^{-2+\varepsilon_{2}} .
$$

\section{Examples 4.3.}

1. In the situation of section $2, \xi_{l}=\sqrt[3]{l^{3}+1}, \theta=\xi_{l}-l \sim \frac{1}{3} l^{-2}$ and $L$ is the projective line of equation

$$
x_{1}+\zeta x_{2}+\zeta^{2} x_{3}=0
$$

The point $\boldsymbol{\beta}=\left(1: \zeta^{-1}: \zeta^{-2}\right)$ has height 1 and thus $c(L)=(6 \sqrt{3})^{1 / 2}$. We have $\theta_{1} \theta_{2} \theta_{3}=1$ and $\left|\theta_{2}\right|=\left|\theta_{3}\right|$. Thus $\|\theta\|=\theta$ and $H_{0}(\theta)=\theta^{-1 / 2}$. Inequality (4.1) becomes $\theta<(6 \sqrt{3})^{-2 / 3} \approx 0.209 \ldots$, which is satisfied for $l \geq 2$. In this case, Theorem 4.1 gives

$$
\begin{aligned}
\mu_{\mathrm{eff}}\left(\xi_{l}\right) & \leq 1+\frac{\frac{3}{2} h_{0}(\theta)+\log c(L)}{\log (1 /\|\theta\|)-\frac{1}{2} h_{0}(\theta)-\log c(L)} \\
& =1+\frac{-\frac{3}{4} \log \left(\xi_{l}-l\right)+\frac{1}{2} \log (6 \sqrt{3})}{-\frac{3}{4} \log \left(\xi_{l}-l\right)-\frac{1}{2} \log (6 \sqrt{3})} \\
& =2+\frac{2 \log (6 \sqrt{3})}{3 \log l}+O\left((\log l)^{-2}\right) \text { for } l \rightarrow+\infty
\end{aligned}
$$


2. Another example is given by irrational cubic numbers of trace 0 , e.g. $\theta=\eta_{b} \sim b^{-1}$ is the real root of $x^{3}+b x-1=0$ for some integer $b \geq 5$. Now $L$ is the projective line of equation

$$
x_{1}+x_{2}+x_{3}=0 .
$$

The same computation shows that

$$
\begin{aligned}
\mu_{\mathrm{eff}}(\theta) & =1+\frac{-\frac{3}{4} \log \left(\eta_{b}\right)+\frac{1}{2} \log (6 \sqrt{3})}{-\frac{3}{4} \log \left(\eta_{b}\right)-\frac{1}{2} \log (6 \sqrt{3})} \\
& =2+\frac{4 \log (6 \sqrt{3})}{3 \log l}+O\left((\log l)^{-2}\right) \text { for } b \rightarrow+\infty .
\end{aligned}
$$

\section{Conjugates lying ON a fiXed Curve.}

We want to generalize Theorem 4.1 replacing $L$ by any fixed projective curve $\mathcal{C}$. To do that, in place of the Beukers-Schlickewei estimate in [4] used above, we need a recent result of Masser and the authors of this article.

Theorem 5.1. Let $\mathcal{C} \subset \mathbb{P}_{r-1}$ be a projective curve defined over $\overline{\mathbb{Q}}$. There exists a positive $c=c(\mathcal{C})$ effectively depending on $\mathcal{C}$ such that the following holds. Let $\boldsymbol{\alpha} \in \mathbb{P}_{r-1}(\overline{\mathbb{Q}}), \boldsymbol{\theta} \in \mathcal{C}(\overline{\mathbb{Q}})$ and choose $K \geq c$ and a natural number $n \geq K$. Then, if

$$
\alpha_{1} \theta_{1}^{n}+\cdots+\alpha_{r} \theta_{r}^{n}=0
$$

and if there are no proper vanishing subsums,

$$
\frac{h(\boldsymbol{\alpha})}{n} \geq \frac{h(\boldsymbol{\theta})}{r-1}-c\left(\frac{1}{K} h(\boldsymbol{\theta})+h(\boldsymbol{\theta})^{1 / 2}+K\right) .
$$

Proof. Let $f_{1}, \ldots, f_{r} \in \overline{\mathbb{Q}}(\mathcal{C})$ be the coordinate functions on $\mathcal{C}$. Then $f_{i} / f_{j}$ is non-constant for some $i \neq j$. We apply [2, Theorem 4.1], taking into account [2, Remark 4.2 i) and ii)].

Replacing in our construction [4], Lemma 2.3, by this theorem with $r=3$ we get the following statement. Theorem 1.1 announced in the introduction immediately follows from it.

Theorem 5.2. Let $\mathbb{K} \subseteq \mathbb{C}$ be a real cubic number; denote by $\sigma_{1}=\mathrm{Id}, \sigma_{2}, \sigma_{3}$ the immersions $\mathbb{K} \hookrightarrow \overline{\mathbb{C}}$. Let $\mathcal{C} \subset \mathbb{P}_{2}$ be a projective curve defined over $\overline{\mathbb{Q}}$ and let $c=c(\mathcal{C})$ be the constant in Theorem 5.1. Let $\hbar>0, \lambda>1 / 2$ and $\varepsilon \in(0,1)$ such that

$$
\hbar \geq c^{2} \min \left(1, \frac{(\lambda-1 / 2)^{2}}{8(\lambda+1)} \varepsilon\right)^{-2} .
$$

Let $\theta \in \mathbb{K}$ such that

$$
h_{0}(\theta) \leq \hbar, \quad \log \|\theta\| \leq-\lambda \hbar .
$$

We assume:

$$
\left(\sigma_{1} \theta: \sigma_{2} \theta: \sigma_{3} \theta\right) \in \mathcal{C}(\overline{\mathbb{Q}}) .
$$

Then

$$
\mu_{\mathrm{eff}}(\xi) \leq \frac{\lambda+1}{\lambda-1 / 2}+\varepsilon
$$

for every generator $\xi$ of $\mathbb{K}$ 
Proof. We argue as in the proof of Theorem 4.1, keeping the same notation. Recall equation (4.5),

$$
\xi_{1}^{\prime} \alpha_{n, 1} \theta_{1}^{n}+\xi_{2}^{\prime} \alpha_{n, 2} \theta_{2}^{n}+\xi_{3}^{\prime} \alpha_{n, 3} \theta_{3}^{n}=0 .
$$

We apply Theorem 5.1 with $r=3$ and with $\boldsymbol{\alpha}$ replaced by $\tilde{\boldsymbol{\alpha}}_{n}:=\left(\xi_{1}^{\prime} \alpha_{n, 1}\right.$ : $\left.\xi_{2}^{\prime} \alpha_{n, 2}: \xi_{3}^{\prime} \alpha_{n, 3}\right)$. Remark that the condition on no proper vanishing subsums is trivially satisfied since $r=3$ (indeed $\xi_{j}^{\prime}, \alpha_{n, j}$ and $\theta_{j}$ are non zero). By this theorem, for any $K \geq c$ and for any integer $n \geq K$,

$$
\begin{aligned}
\frac{1}{n} h\left(\tilde{\boldsymbol{\alpha}}_{n}\right) & \geq \frac{1}{2} h_{0}(\theta)-c\left(\frac{1}{K} h_{0}(\theta)+h_{0}(\theta)^{1 / 2}+K\right) \\
& \geq \frac{1}{2} h_{0}(\theta)-c\left(\frac{1}{K}+\frac{1}{\hbar^{1 / 2}}+\frac{K}{\hbar}\right) \hbar
\end{aligned}
$$

with $c=c(\mathcal{C})$ the constant appearing in that theorem. Let $\varepsilon^{\prime} \in(0,1)$, which will be fixed later, and choose $K=c / \varepsilon^{\prime}$. Then for $\hbar \geq\left(c / \varepsilon^{\prime}\right)^{2}$ and $n \geq c / \varepsilon^{\prime}$,

$$
\frac{1}{n} h\left(\tilde{\boldsymbol{\alpha}}_{n}\right) \geq \frac{1}{2} h_{0}(\theta)-3 \varepsilon^{\prime} \hbar .
$$

By equation (4.3) and since $h\left(\tilde{\boldsymbol{\alpha}}_{n}\right) \leq h\left(\boldsymbol{\xi}^{\prime}\right)+h_{0}\left(\alpha_{n}\right)$, we have

$$
\begin{aligned}
\frac{1}{n} h_{0}\left(\alpha_{n}^{\prime} \theta_{n}\right) & \leq \frac{h\left(\boldsymbol{\xi}^{\prime}\right)}{n}-\frac{1}{n} h\left(\tilde{\boldsymbol{\alpha}}_{n}\right)+2 h_{0}(\theta) \\
\frac{1}{n} \log \left\|\alpha_{n}^{\prime} \theta_{n}\right\| & \leq \frac{h\left(\boldsymbol{\xi}^{\prime}\right)}{n}-\frac{1}{n} h\left(\tilde{\boldsymbol{\alpha}}_{n}\right)+h_{0}(\theta)+\log \|\theta\|
\end{aligned}
$$

Thus, if in addition $n \geq \frac{h\left(\boldsymbol{\xi}^{\prime}\right)}{\varepsilon^{\prime} \hbar}$, we obtain from (5.3) and (5.2)

$$
\frac{1}{n} h_{0}\left(\alpha_{n}^{\prime} \theta_{n}\right) \leq \varepsilon^{\prime} \hbar-\frac{1}{2} h_{0}(\theta)+3 \varepsilon^{\prime} \hbar+2 h_{0}(\theta) \leq\left(\frac{3}{2}+4 \varepsilon^{\prime}\right) \hbar
$$

and

$$
\begin{aligned}
\frac{1}{n} \log \left\|\alpha_{n}^{\prime} \theta_{n}\right\| & \leq \varepsilon^{\prime} \hbar-\frac{1}{2} h_{0}(\theta)+3 \varepsilon^{\prime} \hbar+h_{0}(\theta)-\lambda \hbar \\
& \leq\left(\frac{1}{2}-\lambda+4 \varepsilon^{\prime}\right) \hbar=-\omega\left(\frac{3}{2}+4 \varepsilon^{\prime}\right) \hbar
\end{aligned}
$$

with

$$
\omega=\frac{\lambda-1 / 2-4 \varepsilon^{\prime}}{3 / 2+4 \varepsilon^{\prime}} .
$$

Note that $\omega>0$ if $4 \varepsilon^{\prime}<\lambda-1 / 2$. Assume further $8 \varepsilon^{\prime}<\lambda-1 / 2$. Lemma 3.5 (with $r=3$ and $s=2$ ) then shows that $\xi$ has irrationality measure

$$
\begin{aligned}
\leq 1+1 / \omega & =\frac{\lambda+1}{\lambda-1 / 2}+\frac{4(\lambda+1) \varepsilon^{\prime}}{(\lambda-1 / 2)\left(\lambda-1 / 2-4 \varepsilon^{\prime}\right)} \\
& \leq \frac{\lambda+1}{\lambda-1 / 2}+\frac{8(\lambda+1) \varepsilon^{\prime}}{(\lambda-1 / 2)^{2}} .
\end{aligned}
$$

The result follows on choosing $\varepsilon^{\prime}:=\min \left(1, \frac{(\lambda-1 / 2)^{2}}{8(\lambda+1)} \varepsilon\right) \leq \frac{1}{8}(\lambda-1 / 2)$. 
Example. As an easy example, let $t \in \mathbb{Q}^{*}$ and take for $\theta_{t}$ the root of $x^{3}+x^{2}+t x+t^{6}$ closest to 0 . The projective point $P_{t} \in \mathbb{P}_{2}$ defined by the conjugates of $\theta_{t}$ lies on the projective curve $\left(x_{1} x_{2}+x_{1} x_{3}+x_{2} x_{3}\right)^{6}-\left(x_{1}+x_{2}+\right.$ $\left.x_{3}\right)^{9} x_{1} x_{2} x_{3}=0$ (which is absolutely irreducible and of genus 10 , according to the computer algebra system [11]). Moreover, an easy computation shows that the above conjugates have all absolute value $\leq 1$ for $t \in(0,1]$ and that $\log \left|\theta_{t}\right| \sim 5 \log |t|$ as $t \rightarrow 0$. Let now $t=1 / l$ for a large $l \in \mathbb{N}$. By (3.2), $H_{\text {finite }}\left(\theta_{1 / l}\right)=l^{2}$. Thus

$$
h_{0}\left(\theta_{1 / l}\right) \lesssim 2 \log l, \quad \text { and } \quad \log \left\|\theta_{1 / l}\right\| \sim-3 \log l .
$$

as $l \rightarrow+\infty$. Theorem 5.2 then shows that $\theta_{1 / l}$ has effective irrationality measure $\leq 5 / 2+\varepsilon$ for $l$ large with respect to $1 / \varepsilon$.

Remark 5.3. Let $E$ be an elliptic curve (of rank $>0$ ) and $u, v: E \rightarrow \mathbb{P}_{1}$ be non constant morphisms. It would be tempting to apply Theorem 5.2 to the family of cubic equations $x^{3}+x^{2}+u(P) x+v(P)=0$ parametrized by $P \in E(Q)$, but unfortunately this does not work, as we briefly show. Let $\theta_{P}$ be the root of $x^{3}+x^{2}+u(P) x+v(P)=0$ closest to 0 . Then, by "Weil's Height Machine" and by a conjecture of Lang proved by David and Hirata-Kohno [10, Conjecture 1.2], it is possible to show that $\log \left\|\theta_{P}\right\| \sim$ $\frac{d}{6} \hat{h}(P) \rightarrow+\infty$ with $d=-\operatorname{deg} \operatorname{div}\left(1, u^{3}, v^{2}\right)$, preventing any application of Theorem 5.2.

Back to cubic equations parametrized by rational points on a curve of genus 0 . The example above can be generalized to find an effective upper bound for the irrationality measure of the values of a cubic algebraic function holomorphic at 0 . More precisely, let $g$ be a power series in the variable $t$, with rational coefficients, representing a cubic algebraic function, i.e. $g$ has degree 3 over $\overline{\mathbb{Q}}(t)$. We assume that also the conjugates $g_{1}=g, g_{2}, g_{3}$ of $g$ over $\overline{\mathbb{Q}}(t)$, are represented by analytic functions in the closed disk $|t| \leq e^{-c_{0}}$, for some $c_{0}>0$. We have:

Corollary 5.4. Let $\varepsilon \in(0,1 / 12)$. There exists $C(\varepsilon, g) \geq c_{0} \geq 1$ such that the following holds. Let $a \in \mathbb{Z}, b \in \mathbb{N}$ coprime, with

$$
\log |a| \leq \varepsilon \log b
$$

and

$$
\log b \geq C(\varepsilon, g) .
$$

Then $g(a / b)$ is irrational with effective irrationality measure $\leq 2+13 \varepsilon$.

Proof. Let $t_{0}=a / b$. Then, by (5.4), (5.5) and since $\varepsilon<1 / 2$,

$$
\log \left|t_{0}\right|=\log |a|-\log b \leq-(1-\varepsilon) \log b \leq-(\log b) / 2 \leq-c_{0}
$$

if $C(\varepsilon, g) \geq c_{0}^{2}$. Observe also that $H\left(t_{0}\right)=b$, since $a, b$ are coprime and $\left|t_{0}\right|<1$. We denote by $c_{1}, \ldots, c_{5}$ positive constants depending only on $g$ and on $\varepsilon$.

By the functorial properties of the height, setting $\xi=g\left(t_{0}\right)$, we have

$$
H(\xi) \leq e^{c_{1}} b^{d},
$$

where we may assume $d \geq 1$. 
Let $N=[6 d / \varepsilon]$. We consider a Padé approximant at 0 of order $N$ for $\left(1, g, g^{2}\right)$, i. e. a non-zero vector of polynomials $\left(Q_{0}, Q_{1}, Q_{2}\right) \in \mathbb{Q}[t]$ of degree at most $N$ such that

$$
f=Q_{0}+Q_{1} g+Q_{2} g^{2}
$$

vanishes at 0 with multiplicity $\geq[3(N+1)] \geq 3 N$. This implies

$$
\begin{gathered}
H\left(Q_{0}\left(t_{0}\right): Q_{1}\left(t_{0}\right): Q_{2}\left(t_{0}\right)\right) \leq e^{c_{2}} b^{N} \\
\left|f\left(t_{0}\right)\right| \leq e^{c_{2}}\left|t_{0}\right|^{3 N},
\end{gathered}
$$

where $c_{2}$ depends only on the $Q_{i}$. Moreover $f \neq 0$ since $g$ has degree 3 over $\mathbb{Q}(t)$. Since $f$ is analytic in $D\left(0, e^{-c_{0}}\right)$, there exists $c_{3} \geq c_{0}$ such that $f$ does not vanish for $0<|t| \leq e^{-c_{3}}$. Thus, if $C(\varepsilon, g) \geq c_{3}$ then $\theta:=f\left(t_{0}\right)$ is a non zero algebraic number of degree $\leq 3$. By (5.6), (5.7) and by (3.3) of Lemma 3.4 we have

$$
\begin{aligned}
H_{0}(\theta) & \leq 3 H(\xi)^{6} H\left(Q_{0}\left(t_{0}\right): Q_{1}\left(t_{0}\right): Q_{2}\left(t_{0}\right)\right) \leq e^{c_{4}} b^{(1+\varepsilon) N}, \\
\|\theta\| & \leq H(\xi)^{6} H\left(Q_{0}\left(t_{0}\right): Q_{1}\left(t_{0}\right): Q_{2}\left(t_{0}\right)\right)|\theta| \leq e^{c_{4}} b^{(1+\varepsilon) N}\left|t_{0}\right|^{3 N} .
\end{aligned}
$$

For $j=1,2,3$ let $f_{j}=Q_{0}+Q_{1} g_{j}+Q_{2} g_{j}^{2}$ and $\theta_{j}=f_{j}\left(t_{0}\right)$. We want to apply Theorem 5.2 with $\mathcal{C} \subseteq \mathbb{P}_{2}$ be the projective curve parametrized by $\left(f_{1}: f_{2}: f_{3}\right)$. Let $c>0$ be the constant appearing in that theorem. Set $c_{5}:=\max \left(c_{3}, c_{4} / N, c\right)$ and

Set also

$$
\kappa:=\frac{\log (b /|a|)}{(1+\varepsilon) \log b+c_{5}} .
$$

$$
\hbar:=\left((1+\varepsilon) \log b+c_{5}\right) N>0, \quad \lambda:=3 \kappa-1
$$

and choose $C(\varepsilon, g):=\left(\frac{32 c_{5}}{\varepsilon}\right)^{2}$. Remark that

$$
1-\kappa=\frac{\varepsilon \log b+\log |a|+c_{5}}{(1+\varepsilon) \log b+c_{5}} \leq 3 \varepsilon .
$$

by (5.4) and (5.5). In particular

$$
\kappa \geq 1-3 \varepsilon \geq 3 / 4
$$

since $\varepsilon \leq 1 / 12$. This implies

$$
\frac{(\lambda-1 / 2)^{2}}{8(\lambda+1)} \varepsilon=\frac{3(\kappa-1 / 2)^{2}}{8 \kappa} \varepsilon \geq \frac{\varepsilon}{32} .
$$

Thus

$$
c^{2} \min \left(1, \frac{(\lambda-1 / 2)^{2}}{8(\lambda+1)} \varepsilon\right)^{-2} \leq\left(\frac{32 c_{5}}{\varepsilon}\right)^{2}=C(\varepsilon, g) \leq \log b \leq \hbar
$$

by (5.5). Assumptions (5.1) is satisfied. Moreover, by (5.8) we have $h_{0}(\theta) \leq$ $\hbar$ and

$$
-\frac{\log \|\theta\|}{\hbar} \geq \frac{3 N \log (b /|a|)-(1+\varepsilon) N \log b-c_{5} N}{\left((1+\varepsilon) \log b+c_{5}\right) N}=3 \kappa-1=\lambda .
$$

Thus (5.2) is also satisfied.

By Theorem 5.2, $\theta$ is a cubic irrational and every generator of $\mathbb{Q}(\theta)$ has irrationality measure

$$
\leq \frac{\lambda+1}{\lambda-1 / 2}+\varepsilon=\frac{\kappa}{\kappa-1 / 2}+\varepsilon=2+\frac{1-\kappa}{\kappa-1 / 2}+\varepsilon \leq 2+13 \varepsilon
$$


where we have plugged in (5.9) and (5.10) into the computation.

We finally remark that $\xi$ is a generator of $\mathbb{Q}(\theta)$, since $\mathbb{Q}(\theta) \subseteq \mathbb{Q}(\xi)$ and $[\mathbb{Q}(\xi): \mathbb{Q}] \leq 3=[\mathbb{Q}(\theta): \mathbb{Q}]$.

Let $g$ be an arbitrary algebraic function (of degree possibly $>3$ ). In $[9$, Theorem 1], Chudnovski announced the upper bound $\mu_{\text {eff }}(g(a / b)) \leq 2+\varepsilon$ for every $\varepsilon>0$ provided that $b^{\varepsilon} \geq c_{1}(\varepsilon, g)|a|^{2+\varepsilon}$. The Author presents a complete proof only in the special case of a cubic algebraic function; his proof rests on the well-known fact that cubic algebraic functions satisfies a Riccati equation. A standard computation, starting from the choice of the parameter $N$ at the end of the proof of his Theorem 1, shows that his method is indeed able to provide a more explicit result:

$$
\begin{aligned}
\mu_{\mathrm{eff}}(g(a / b)) \leq 2+\frac{2 c(g)}{\varepsilon \sqrt{\log b}}+ & \frac{2}{\varepsilon} \cdot \frac{\log |a|}{\log b}, \\
& \text { if }|a| \leq b^{1 / 2-\varepsilon} \text { and } \log b \geq 4 c(g)^{2} / \varepsilon^{2} .
\end{aligned}
$$

Our method (at the price of an explicit computation of the Padé approximation $f$ and of a more precise zero's lemma) can provide an estimate of a similar shape:

$$
\begin{aligned}
\mu_{\mathrm{eff}}(g(a / b)) \leq 2+\frac{9}{\varepsilon} \sqrt{\frac{c(g)}{\log b}}+\frac{2}{\varepsilon} \cdot \frac{\log |a|}{\log b} & \\
& \text { if }|a| \leq b^{1 / 2-\varepsilon} \text { and } \log b \geq 2^{10} c(g)^{3} / \varepsilon^{4} .
\end{aligned}
$$

In this context, it is not out of place to mention that Zudilin [14] obtained a general statement on the values of $G$-functions, and thus in particular applying to algebraic functions. A very special case of his result gives

$$
\mu_{\mathrm{eff}}(g(a / b)) \leq 2+c(g)\left(\frac{1}{(\log b)^{1 / 3}}+\frac{\log |a|}{\log b}\right),
$$

for an algebraic function $g$ (of arbitrary large degree) and a rational $a / b$, provided that $\log |a|<\frac{1}{2} \log b-c(g)(\log b)^{2 / 3}$ and $\log b>c(g)$. This is a weaker version of $(5.11)$ (essentially $(\log b)^{1 / 3}$ instead of $\sqrt{\log b}$ ), which is not surprising, since his estimate is a specialization of a more general result.

\section{REFERENCES}

[1] K. Alladi and M. L. Robinson, "Legendre polynomials and irrationality." $J$. Reine Angew. Math. 318 (1980), 137-155.

[2] F. Amoroso, D. Masser and U. Zannier, "Bounded Height Problems and Silverman Specialization Theorem" , Duke Math J., 166, no. 13 (2017), 2599-2642.

[3] A. Baker, "Rational approximations to certain algebraic numbers". Proc. London Math. Soc. (3) 14 385-398 (1964).

[4] F. Beukers and F. Schlickewei, "The equation $\mathrm{x}+\mathrm{y}=1$ in finitely generated groups". Acta Arithmetica. 78 (1996), no. 2, 189-199.

[5] F. Beukers and R. Tijdeman, "On the multiplicities of binary complex recurrences". Compositio Math. 51 (1984), no. 2, 193-213.

[6] E. Bombieri, "On the Thue-Siegel-Dyson theorem". Acta Math. 148 (1982), 255-296. 
[7] E. Bombieri and P. Cohen, "Siegel's lemma, Padé approximations and jacobians". Ann. Scuola Norm. Sup. Pise Cl. Sci. (5), 25 (1997), no. 1-2, $155-178$.

[8] Y. Bugeaud "Effective irrationality measures for real and p-adic roots of rational numbers close to 1 , with an application to parametric families of Thue-Mahler equations". Math. Proc. Cambridge Philos. Soc. 164 (2018), no. 1, 99-108.

[9] G. V. Chudnovsky, "The Thue-Siegel-Roth theorem for values of algebraic functions". Proc. Japan Acad. Ser. A Math. Sci. 59 (1983), no. 6, 281-284.

[10] S. David and N. Hirata-Kohno, "Linear forms in elliptic logarithms". J. Reine Angew. Math. 628 (2009), 37-89.

[11] W. Decker, G.-M. Greuel, G. Pfister and H. Schönemann, "SinguLAR 4-1-1 - A computer algebra system for polynomial computations." http://www.singular.uni-kl.de (2018).

[12] N. I. Feldman, "An effective power sharpening of a theorem of Liouville". Izv. Akad. Nauk SSSR Ser. Mat. 35 (1971) 973-990.

[13] M. Hata, "Legendre type polynomials and irrationality measures". J. Reine Angew. Math. 407 (1990), 99-125.

[14] V. V. Zudilin, "On the irrationality measure of values of G-functions". Izv. Ross. Akad. Nauk Ser. Mat. 60 (1996), no. 1, 87-114; translation in Izv. Math. 60 (1996), no. 1, 91-118 